\title{
Lipooligosaccharide locus classes and putative virulence genes among chicken and human Campylobacter jejuni isolates
}

\author{
Patrik Ellström ${ }^{1,2^{*}}$, Ingrid Hansson ${ }^{3}$, Anna Nilsson ${ }^{1}$, Hilpi Rautelin ${ }^{1,4}$ and Eva Olsson Engvall ${ }^{3}$
}

\begin{abstract}
Background: Campylobacter cause morbidity and considerable economic loss due to hospitalization and post infectious sequelae such as reactive arthritis, Guillain Barré- and Miller Fischer syndromes. Such sequelae have been linked to C. jejuni harboring sialic acid structures in their lipooligosaccharide (LOS) layer of the cell wall. Poultry is an important source of human Campylobacter infections but little is known about the prevalence of sialylated C. jejuni isolates and the extent of transmission of such isolates to humans.

Results: Genotypes of $C$. jejuni isolates from enteritis patients were compared with those of broiler chicken with pulsed-field gel electrophoresis (PFGE), to study the patterns of LOS biosynthesis genes and other virulence associated genes and to what extent these occur among Campylobacter genotypes found both in humans and chickens. Chicken and human isolates generally had similar distributions of the putative virulence genes and LOS locus classes studied. However, there were significant differences regarding LOS locus class of PFGE types that were overlapping between chicken and human isolates and those that were distinct to each source.
\end{abstract}

Conclusions: The study highlights the prevalence of virulence associated genes among Campylobacter isolates from humans and chickens and suggests possible patterns of transmission between the two species.

Keywords: Campylobacter, LOS, Pulsed Field Gel Electrophoresis (PFGE), Virulence genes, Transmission

\section{Background}

Campylobacter is the most common cause of bacterial enteritis in the western world accounting for more than 200000 reported human infections per year in the EU [1]. Campylobacter jejuni and C. coli are the two most common species infecting humans. The disease is normally self-limiting and resolves within 2-3 weeks. However, in some cases patients need to be hospitalized due to severe enteritis or to sequelae such as reactive arthritis, Guillain Barré- or Miller Fischer syndromes [2-5]. The total economic burden of campylobacteriosis has been estimated to appr. 2.4 billion euros annually in the EU [6]. Campylobacter is a zoonotic pathogen and the

\footnotetext{
* Correspondence: patrik.ellstrom@medsci.uu.se

'Department of Medical Sciences, Clinical Microbiology, Uppsala University, SE-75185 Uppsala, Sweden

${ }^{2}$ Department of Medical Biochemistry and Microbiology, Zoonosis Science Center, Uppsala University, BMC A9:3, Husargatan 3, SE-75123 Uppsala, Sweden

Full list of author information is available at the end of the article
}

sources of infection mainly include products from farm animals and contaminated water. In many countries, poultry is the single most important source with broiler meat estimated to account for $20-30 \%$ of human cases in the EU [6]. The prevalence of Campylobacter generally shows a strong seasonal variation in European countries, with a peak between June and September, both in terms of reported human cases and positive chicken flocks [1]. The mechanisms behind the pathogenicity of C. jejuni are poorly understood. The existence of virulence associated genes has been proposed in several studies but few have been correlated to disease severity in clinical materials $[2,4,5,7-10]$. The structure of the lipooligosaccharide (LOS) layer of $C$. jejuni has been suggested to be an important determinant of the pathogenicity of certain postinfectious sequelae $[4,5,10]$. The synthesis of LOS in the outer membrane of $C$. jejuni is regulated by the LOS operon and based on the genomic organization, this operon has been categorized into 19 different LOS locus classes denoted A to $\mathrm{S}[11,12]$. 
Strains belonging to LOS locus class A, B and C harbor genes that enable sialylation of the LOS structures. Such strains have been associated with the post-infectious sequelae Guillain Barré- and Miller Fischer syndromes as well as with reactive arthritis $[4,5,10]$. Some studies have also proposed that $C$. jejuni strains with sialylated LOS might be involved in more severe symptoms of enteritis as well as invasive infection [5, 13, 14]. However in previous studies, we have not been able to find any associations between such strains and severe enteritis or with invasive infection in patients $[7,8]$. The prevalence of $C$. jejuni strains with genes for sialylation of LOS among enteritis patients seems to vary between 50 and $60 \%[5,8,15]$. Although poultry is an important source of Campylobacter infection in many countries, very little is known about the distribution of LOS locus classes among $C$. jejuni from broiler flocks reared for consumption $[15,16]$. In the present study, we compared isolates from Swedish enteritis patients with domestically acquired C. jejuni infection to $C$. jejuni isolates from broiler chickens raised in Sweden, with the aim to study patterns of virulence associated genes and their possible involvement in transmission from chickens to humans in Sweden.

\section{Results}

\section{Species identification and PFGE typing}

A total of $163 \mathrm{C}$. jejuni isolates were included in the study, including 104 from chicken and 59 from humans. Pulsed field gel electrophoresis (PFGE) showed that the 163 C. jejuni isolates fell into 66 different SmaI types. Of these, 19 types were unique for humans ( 24 isolates) and 32 types were unique for chicken (43 isolates). Fifteen SmaI types were detected among isolates of both human (32 isolates) and chicken (57 isolates) origin. The unique SmaI types each contained from 1 up to 3 human isolates or from 1 up to 4 chicken isolates. The shared SmaI types contained from 1 up to 7 human isolates and from 1 up to 19 chicken isolates. Seven isolates (3 from humans and 4 from chickens) were not cleaved by SmaI and hence, the SmaI type could not be determined. In total, $55 \%$ of all isolates belonged to SmaI types that were shared between human and chicken isolates.

\section{Lipooligosaccharide (LOS) locus classes}

All isolates were screened for LOS locus class by PCR. In general the distribution of LOS classes were similar among the human and chicken isolates. LOS locus class $\mathrm{C}$ was the most common in both materials followed by class $\mathrm{H}$. The LOS locus classes with genes for incorporation of sialic acids in the LOS (classes A, B and C) together constituted 57 and $50 \%$ of the human and chicken isolates respectively (Table 1 ). Temporal variations in the occurrence of the different LOS classes were
Table 1 Distribution of LOS locus classes among the human and chicken C. jejuni isolates

\begin{tabular}{lll}
\hline LOS locus class & Human isolates & Chicken isolates \\
\hline $\mathrm{A}^{\mathrm{a}}$ & $2(3 \%)$ & $1(1 \%)$ \\
$\mathrm{B}^{\mathrm{a}}$ & $7(12 \%)$ & $9(9 \%)$ \\
$\mathrm{AB}^{\mathrm{a}}$ & 0 & $2(2 \%)$ \\
$\mathrm{C}^{\mathrm{a}}$ & $25(42 \%)$ & $40(38 \%)$ \\
$\mathrm{E}$ & $5(8 \%)$ & $11(11 \%)$ \\
$\mathrm{H}$ & $12(20 \%)$ & $17(16 \%)$ \\
$18 d f^{b}$ & $6(10 \%)$ & $17(16 \%)$ \\
Untypeable & $2(3 \%)$ & $13(13 \%)$ \\
Total & 59 & 104 \\
\hline
\end{tabular}

aOS locus classes enabling sialylation of LOS

${ }^{\mathrm{b}}$ Orf18df indicates isolates of the nonsialylated classes $\mathrm{D}, \mathrm{F}, \mathrm{I}, \mathrm{J}, \mathrm{K}, \mathrm{N}, \mathrm{S}$, or Q

analyzed but no apparent seasonal differences were observed among either human or chicken isolates.

As expected, there was generally a strong coherence between LOS locus class and SmaI type where the vast majority of the isolates in one particular SmaI type had the same LOS locus class (data not shown). To study if certain LOS locus classes were connected to the presumed chicken source of the human isolates in this material, we analyzed the association of LOS locus class with isolates that were shared between human and chicken isolates and those that were unique to the respective source in this material. All SmaI types with more than 2 isolates, in total 26 (11 unique and 15 shared) were included in the comparison, covering altogether 123 isolates (44 human and 79 chicken). Among isolates with LOS locus class $C$ there were no significant differences in the proportions belonging to the shared and the unique SmaI types $(P=0.120$, Table 2). Isolates with LOS locus class $\mathrm{H}$ were significantly more common among SmaI types that were shared among chicken and humans than among SmaI types that were unique to each species $(P=0.014)$. LOS

Table 2 LOS locus classes of C. jejuni from humans and chickens according to shared or unique Smal types

\begin{tabular}{llll}
\hline LOS locus class & Shared Smal types & Unique Smal types & $P$ value \\
\hline $\mathrm{A}^{\mathrm{a}}$ & 1 & 0 & 1.000 \\
$\mathrm{~B}^{\mathrm{a}}$ & 3 & 7 & 0.0009 \\
$\mathrm{C}^{\mathrm{a}}$ & 43 & 7 & 0.120 \\
$\mathrm{E}$ & 13 & 1 & 0.300 \\
$\mathrm{H}$ & 25 & 1 & 0.014 \\
$\mathrm{E}+\mathrm{H}$ & 38 & 2 & 0.001 \\
18df & 7 & 11 & $<0.0001$ \\
Untypeable & 4 & 0 & 0.575 \\
Total & 96 & 27 & \\
\hline
\end{tabular}

aOOS locus classes enabling sialylation of LOS

${ }^{\mathrm{b}}$ Orf18df indicates isolates of the nonsialylated classes D, F, I, J, K, N, S, or Q 
locus class $\mathrm{E}$ and $\mathrm{H}$ are genetically closely related [11] and an analysis of these two classes as one group showed a highly significant overrepresentation of such isolates among the shared SmaI types $(P=0.001)$. On the other hand, isolates with LOS locus class B were more common among SmaI types that were unique for chickens and humans, as compared to shared SmaI types $(P=0.0009)$. This was true also for the ORF18 positive isolates $(P<0.0001)$.

\section{Putative virulence genes}

The prevalences of the putative virulence genes were generally similar between the human and chicken isolates (Table 3). The gene fucP was detected in $64 \%$ of the human- and $55 \%$ of the chicken isolates respectively $(P=0.232)$, whereas virB11 was not found at all among the human isolates and only in $11 \%$ of the tested chicken isolates. The genes ceuE, pldA and ciaB were present in the vast majority of both human and chicken isolates. We analyzed if the presence of these genes in the $C$. jejuni isolates was related to season but no significant seasonal variation was found. The largest difference was found between $f u c P$ positive vs $f u c P$ negative among human isolates in June $(P=0.281)$. As for the LOS locus classes, there was strong coherence between the putative virulence genes and SmaI type (data not shown). Furthermore, the presence of the gene fucP was strongly associated with certain LOS locus classes, both in the human and chicken isolates (Table 4). In particular, isolates of LOS locus class $C$ were almost exclusively fucP positive, whereas nearly all isolates of LOS locus classes $\mathrm{E}$ and $\mathrm{H}$ were $f u c P$ negative. Isolates with LOS locus classes A and B were generally fucP positive.

\section{Discussion}

In this study on human and chicken $C$. jejuni isolates obtained during year 2009 and analyzed by PFGE, we found that the majority of isolates (55\%) had SmaI types that were shared between human and chicken isolates. Assuming that this is a result of transmission between the two species, it supports the current notion that

Table 3 Distribution of the putative virulence genes studied among human and chicken C. jejuni isolates

\begin{tabular}{lll}
\hline Gene & Human isolates & Chicken isolates \\
\hline fucP & $38(64 \%)$ & $57(55 \%)$ \\
CeuE & $58(98 \%)$ & $104(100 \%)$ \\
pldA & $58(98 \%)$ & $39 / 45(87 \%)^{\mathrm{a}}$ \\
CiaB & $59(100 \%)$ & $45 / 45(100 \%)^{\mathrm{a}}$ \\
virB11 & $0(0 \%)$ & $5 / 45(11 \%)^{\mathrm{a}}$ \\
Total & 59 & 104 \\
\hline
\end{tabular}

${ }^{\mathrm{a}}$ Only 45 of the 104 chicken isolates were tested for the presence of these genes
Table 4 Human and chicken isolates of C. jejuni according to LOS locus class and presence of the gene fuc $P$

\begin{tabular}{llll}
\hline LOS locus class & fucP positive & fucP negative & $P$ value \\
\hline $\mathrm{A}^{\mathrm{a}}$ & 3 & 0 & NS \\
$\mathrm{B}^{\mathrm{a}}$ & 12 & 4 & $\mathrm{NS}$ \\
$\mathrm{C}^{\mathrm{a}}$ & 62 & 3 & $P<0.0001$ \\
$\mathrm{E}$ & 0 & 16 & $P<0.0001$ \\
$\mathrm{H}$ & 2 & 27 & $P<0.0001$ \\
Total & 95 & 68 & \\
\hline
\end{tabular}

aOOS locus classes enabling sialylation of LOS

chicken is a major source of human C. jejuni infections. Interestingly, we found that the number of SmaI types that were unique either to chickens $(n=32)$ or to humans $(n=19)$ were higher than the number of SmaI types that were shared between chickens and humans $(n=15)$. This could suggest that there are only certain genotypes among the Swedish chicken isolates that are more likely to be transmitted to humans.

When studying the distribution of LOS locus classes as well as the presence of the putative virulence genes fucP, ceuE, virB11, pldA and ciaB in the human and chicken isolates it was found that the LOS locus classes showed similar distributions among the human and chicken isolates. The LOS locus class $\mathrm{C}$ was the most common in both sources followed by the classes $\mathrm{E}$ and $\mathrm{H}$, which is in line with the results from other studies on human isolates $[5,8,15]$. By a combined analysis of LOS locus classes and the SmaI restriction patterns, we sought to identify potential differences in transmission of isolates with different LOS locus classes between the Swedish chickens and humans as well as from unknown sources. As the chicken isolates in this study represented approximately $99 \%$ of all chickens that were produced in Sweden during 2009, and the human isolates represent all patients in a specific region that have sought medical care for gastrointestinal symptoms attributed to domestically acquired $C$. jejuni infection, it could be assumed that a human $C$. jejuni isolate with a SmaI pattern identical to a chicken isolate most likely actually originated from this chicken source. Human or chicken isolates with SmaI patterns that were unique to either source on the other hand, could be assumed to originate from other sources including environmental, other farm animals or imported chicken. With this analysis we found that isolates of LOS locus class $C$ were equally represented among isolates with SmaI types that were shared between the Swedish chickens and humans as among those that were unique, suggesting that such isolates are readily transmitted between the Swedish chickens and humans, but were also acquired from other sources. Isolates of LOS locus class $\mathrm{E}$ and $\mathrm{H}$ were significantly more likely to have shared SmaI patterns than 
unique ones, suggesting that such isolates are to a high extent spread between Swedish chickens and humans. Isolates with LOS locus class B on the other hand showed an inversed distribution with significantly more isolates having SmaI patterns unique to either chickens or humans, suggesting that such isolates were rarely spread between the two species in this material. The same was true for the ORF 18 positive isolates although this group does not represent a single LOS locus class.

In line with the results from previous studies, we found that nearly all isolates, both from humans and chickens had the gene $c e u E$, suggesting that this gene is important both for human infection and colonization of chickens. The ability of $C$. jejuni isolates to utilize Lfucose, mediated in part by the fucose permease gene fucP, has been suggested to provide an advantage in the colonization of chickens and piglets $[17,18]$. In this material however, the prevalence of $f u c P$ was only slightly higher than $50 \%$ among the human and chicken isolates. In our previous studies we found this particular gene to be present in $49 \%$ of isolates from enteritis patients and in $23 \%$ of isolates from bacteremia patients in Finland $[2,8]$. Together these results suggest that Lfucose utilization by fucP hardly is a prerequisite for colonization of chickens or for causing infections in humans.

\section{Conclusions}

In conclusion, we found that the prevalences of the different LOS locus classes in general, and of the putative virulence factors fucP, ceuE, pldA and ciaB were similar among human and chicken $C$. jejuni isolates in Sweden. However, LOS locus classes $\mathrm{E}$ and $\mathrm{H}$ were more abundant among isolates with SmaI types that were shared between Swedish chickens and humans whereas LOS locus class B was more abundant among isolates with SmaI types unique to the sources. Altogether, these results suggest that humans might acquire $C$. jejuni isolates with different LOS locus classes from different sources. Taking into account the association between $C$. jejuni isolates with some LOS locus classes and post infectious sequelae such as Guillain Barré syndrome and reactive arthritis in humans, this is an interesting finding that needs further studies in larger materials.

\section{Methods}

\section{Bacterial isolates}

Human isolates of domestic origin (as determined from the travel history of the patients) during $2009(N=60)$ were obtained at the Clinical Microbiology Laboratory at Uppsala University Hospital. Chicken isolates were collected within the frame of the Swedish Campylobacter Monitoring Programme for broilers. This has been run by the Swedish Poultry Meat Association since 1991
[19]. In the programme, which includes $99 \%$ of broilers in Sweden, samples were taken from every broiler flock at slaughter. Ten caecal samples were pooled into one composite sample from each flock. In 2009, more than 3000 chicken flocks were sampled and approximately $12 \%$ were positive for Campylobacter. One isolate from every second positive chicken flock was selected for the study, making up a total of 110 isolates from 52 different broiler producers. Preliminary species identification was performed by microscopic examination of morphology and motility as well as oxidase, catalase and hippurate test $[20,21]$.

All isolates were then identified to species level by PCR [22-24]. For this, DNA was extracted using the Qiagen blood and tissue kit (Qiagen, Sollentuna, Sweden) according to the manufacturer's instructions. The following primer pairs were used: MDmapA1 upper- and MDmapA2 lower primers targeting the mapA gene of $C$. jejuni (annealing temperature (AT) $=59{ }^{\circ} \mathrm{C}$ ) [22] as well as the COL3 upper- and MDCOL2 lower primers targeting the ceuE gene of C. coli $\left(\mathrm{AT}=59^{\circ} \mathrm{C}\right)[22,23]$. Primer pair: $\mathrm{C} 412 \mathrm{~F}$ and $\mathrm{C} 1228 \mathrm{R}\left(\mathrm{AT}=58{ }^{\circ} \mathrm{C}\right.$ ) targeting a region of the $16 \mathrm{~S}$ rRNA gene specific for the genus Campylobacter was used to confirm successful DNA extraction [24]. All primers were purchased from Eurogentec (Ougrée, Belgium). Cycling conditions were $10 \mathrm{~min}$ at $95{ }^{\circ} \mathrm{C}, 25 \mathrm{cy}-$ cles of $30 \mathrm{~s}$ at $95^{\circ} \mathrm{C}, 90 \mathrm{~s}$ at annealing temp. and $1 \mathrm{~min}$ at $72{ }^{\circ} \mathrm{C}$, with a final extension step of $10 \mathrm{~min}$ at $72{ }^{\circ} \mathrm{C}$. The strains C. jejuni NCTC11168 (ATCC 700819), C. jejuni 81176 (ATCC BAA-2151) and C. coli LMG6440 were used as positive controls and omission of template was used as negative control. Seven isolates (1 human and 6 chicken) were determined as $C$. coli and excluded from the study.

\section{Genotyping of $C$. jejuni by Pulsed field Gel Electrophoresis (PFGE)}

All isolates were analyzed by PFGE according to standard protocols. The restriction enzyme SmaI was used for cleavage of whole genomic DNA obtained from $C$. jejuni cultured on blood agar plates. For electrophoresis the instrument CHEF- DR II (BioRad, Stockholm, Sweden) was used. The BioNumerics program version 7.5 (Applied Maths, Kortrijk, Belgium) was used for analysis of restriction patterns of the isolates. Isolates with identical restriction patterns were designated as belonging to the same SmaI type.

\section{Putative virulence associated genes}

All C. jejuni isolates were analyzed by PCR for the presence of genes encoding the putative virulence factors, fucose permease $(f u c P)$ and the iron transport protein $(c e u E)$. Furthermore all human isolates and 45 chicken isolates were screened for presence of the genes 
phospholipase (pldA), the Campylobacter invasion antigen $(c i a B)$ and the plasmid borne virulence gene (virB11). A close to $100 \%$ prevalence of the genes pldA and ciaB as well as very low prevalence of $\operatorname{virB11}$ were expected among the chicken isolates from previous studies, and hence only a subset was selected to verify this [25]. The reaction mixture was 1x AmpliTaq Gold 360 buffer with $1.25 \mathrm{U}$ of AmpliTaq Gold 360 polymerase (Applied Biosystems, Austin, USA), $200 \mu \mathrm{M}$ dNTP (Fermentas, St. LeonRot, Germany), $0.2 \mu \mathrm{M}$ of each primer (Eurogentec, Ougrée, Belgium) and $5 \mu$ of template DNA in a total volume of $25 \mu \mathrm{l}$. Cycling conditions were $95{ }^{\circ} \mathrm{C}$ for $10 \mathrm{~min}$ followed by 25 cycles of $95^{\circ} \mathrm{C}$ for 30s, annealing temperatures (fucP: $58{ }^{\circ} \mathrm{C}$, ceuE: $60{ }^{\circ} \mathrm{C}$, pldA: $45{ }^{\circ} \mathrm{C}$, ciaB: $58{ }^{\circ} \mathrm{C}$, virB11: $53{ }^{\circ} \mathrm{C}$ ) for 30 s and $72{ }^{\circ} \mathrm{C}$ for 60 s. For virB11 a touch down protocol was run with 5 cycles at $53{ }^{\circ} \mathrm{C}, 5 \mathrm{cy}-$ cles at $52{ }^{\circ} \mathrm{C}$ and 15 cycles at $51^{\circ} \mathrm{C}$. All reactions ended with an extension step at $72{ }^{\circ} \mathrm{C}$ for $7 \mathrm{~min}$. C. jejuni NCTC 11168 and $C$. jejuni 81176 were used as positive controls and omission of template was used as negative control.

\section{Determination of LOS locus class}

LOS locus class was determined for all isolates by PCR screening as described by Parker et al. [11, 12]. Primer names and sequences were as described in [12]. Amplification of the open reading frame (orf) 12 (waaV) was used to verify successful DNA extraction. The LOS locus classes A and B were identified by the presence of orf7ab (cstII), orf6ab1 (cgtB-1), orf6ab2 (cgtB-2) and orf5bII (cgtA2). Class $C$ was identified by the presence of orf6c (cgtB) and orf7c (cstIII). The classes E, $\mathrm{H}, \mathrm{O}$ and $\mathrm{P}$ were identified by the presence of orf26e and orf27e. The orf18df, present in isolates of the nonsialylated classes D, F, I, J, K, N, S, or Q was used for isolates that were untypeable with the primers above. Cycling conditions were $95{ }^{\circ} \mathrm{C}$ for $10 \mathrm{~min}$ followed by 30 cycles of 30s at $94{ }^{\circ} \mathrm{C}$, 30s at $52{ }^{\circ} \mathrm{C}$, and 60 s at $72{ }^{\circ} \mathrm{C}$ and final extension at $72{ }^{\circ} \mathrm{C}$ for $7 \mathrm{~min}$. Primers for orf6ab1 and orf6ab2 were run together in a duplex PCR with an annealing temperature of $59^{\circ} \mathrm{C}$.

\section{Statistical analysis}

Statistical analyses were performed with GraphPad Prism version 5 (GraphPad Software, San Diego, CA, USA) The $\chi^{2}$ or Fisher's exact tests were used for comparison of categorical data. A $P$ value $<0.05$ was considered significant.

\footnotetext{
Abbreviations

AT, Annealing temperature; ceuE, Gene encoding an iron transport protein; cgtB, $\beta$-1,3-galactosyltransferase; ciaB, Campylobacter invasion antigen; cstll, cstIII, Sialyltransferases; fucP, Fucose permease; LOS, Lipooligosaccharide; ORF, Open reading frame; PFGE, Pulsed field gel electrophoresis; pldA, phospholipase gene; Smal, Restriction enzyme from Serratia marcescens; U, Units; virB11, Plasmid borne virulence gene; waaV, Putative glycosyltransferase
}

\section{Acknowledgements}

The skilled technical assistance of Boel Harbom and Ninni Pudas is gratefully acknowledged, as well as assignment of human infections of domestic origin by Bodil Ardung.

\section{Funding}

This work was financially supported by grants from Stiftelsen Lantbruksforskning [grant number: H0943304], the Swedish Research Council [grant number: 521-2011-3527] and the Swedish Research Council FORMAS [grant number: 221-2012-1442]. The funding sources had no involvement in any part of the study.

\section{Availability of data and materials}

All the data supporting the conclusions is contained within the manuscript or will be shared upon request.

\section{Authors' contributions}

PE planned the studies, analyzed the data and drafted the manuscript, $\mathrm{IH}$ planned the studies and collected the chicken material, AN performed isolate culture and PCR analysis, HR planned the studies, selected the patient isolates and contributed resources, EOE planned the studies, collected the chicken material, analyzed PFGE data and contributed resources. All authors participated in writing the manuscript. All authors read and approved the final manuscript.

\section{Competing interests}

The authors declare that they have no competing interests.

\section{Consent for publication}

Not applicable.

\section{Ethics approval and consent to participate}

The clinical C. jejuni isolates of domestic origin were selected from the strain collection of the Clinical Microbiology Laboratory at Uppsala University Hospital, and the data were analyzed anonymously. No ethical permission was needed. Samples from broilers were taken at slaughter and therefore no ethical permission was required.

\section{Author details}

${ }^{1}$ Department of Medical Sciences, Clinical Microbiology, Uppsala University, SE-75185 Uppsala, Sweden. ${ }^{2}$ Department of Medical Biochemistry and Microbiology, Zoonosis Science Center, Uppsala University, BMC A9:3, Husargatan 3, SE-75123 Uppsala, Sweden. ${ }^{3}$ Department of Microbiology, EU Reference Laboratory for Campylobacter, National Veterinary Institute, SE-75189 Uppsala, Sweden. ${ }^{4}$ Department of Bacteriology and Immunology, University of Helsinki, P.O. Box 21, FIN-00014 Helsinki, Finland.

Received: 19 November 2015 Accepted: 10 June 2016

Published online: 21 November 2016

\section{References}

1. EFSA, ECDC (European Centre for Disease Prevention and Control). The European Union summary report on trends and sources of zoonoses, zoonotic agents and food-borne outbreaks in 2013. EFSA J. 2015;13:3991-4155.

2. Feodoroff $B$, Ellstrom $P$, Hyytiainen $H$, Sarna $S$, Hanninen ML, Rautelin $H$. Campylobacter jejuni isolates in Finnish patients differ according to the origin of infection. Gut pathog. 2010;2:22-9.

3. Feodoroff B, Lauhio A, Ellström P, Rautelin H. A Nationwide Study of Campylobacter jejuni and Campylobacter coli bacteremia in Finland over a 10-year period, 1998-2007, with special reference to clinical characteristics and antimicrobial susceptibility. Clin Infect Dis. 2011;53:e99-106.

4. Godschalk PC, Heikema AP, Gilbert M, Komagamine T, Ang CW, Glerum J, et al. The crucial role of Campylobacter jejuni genes in anti-ganglioside antibody induction in Guillain-Barre syndrome. J Clin Invest. 2004;114:1659-65

5. Mortensen NP, Kuijf ML, Ang CW, Schiellerup P, Krogfelt KA, Jacobs BC, et al. Sialylation of Campylobacter jejuni lipo-oligosaccharides is associated with severe gastro-enteritis and reactive arthritis. Microbes Infect. 2009;11:988-94. 
6. EFSA, ECDC (European Centre for Disease Prevention and Control). Scientific Opinion on Campylobacter in broiler meat production: control options and performance objectives and/or targets at different stages of the food chain. EFSA J. 2011:9:2105-245.

7. Ellstrom P, Feodoroff B, Hanninen ML, Rautelin H. Lipooligosaccharide locus class of Campylobacter jejuni: sialylation is not needed for invasive infection. Clin Microbiol Infect. 2014;20:524-9.

8. Ellström $\mathrm{P}$, Feodoroff $\mathrm{B}$, Hänninen ML, Rautelin $\mathrm{H}$. Characterization of clinical Campylobacter jejuni isolates with special emphasis on lipooligosaccharide locus class, putative virulence factors and host response. Int J Med Microbiol. 2013;303:134-9.

9. Feodoroff B, de Haan CP, Ellstrom P, Sarna S, Hanninen ML, Rautelin H. Clonal distribution and virulence of Campylobacter jejuni isolates in blood. Emerg Infect Dis. 2013;19:1653-5.

10. Koga M, Gilbert M, Takahashi M, Li J, Koike S, Hirata K, et al. Comprehensive analysis of bacterial risk factors for the development of Guillain-Barre syndrome after Campylobacter jejuni enteritis. J Infect Dis. 2006;193:547-55.

11. Parker CT, Gilbert M, Yuki N, Endtz HP, Mandrell RE. Characterization of lipooligosaccharide-biosynthetic loci of Campylobacter jejuni reveals new lipooligosaccharide classes: evidence of mosaic organizations. J Bacteriol. 2008;190:5681-9.

12. Parker $C T$, Horn ST, Gilbert M, Miller WG, Woodward DL, Mandrell RE. Comparison of Campylobacter jejuni lipooligosaccharide biosynthesis loci from a variety of sources. J Clin Microbiol. 2005;43:2771-81.

13. Louwen R, Heikema A, van Belkum A, Ott A, Gilbert M, Ang W, et al. The sialylated lipooligosaccharide outer core in Campylobacter jejuni is an important determinant for epithelial cell invasion. Infect Immun. 2008;76:4431-8.

14. Louwen R, Nieuwenhuis EE, van Marrewijk L, Horst-Kreft D, de Ruiter L, Heikema AP, et al. Campylobacter jejuni translocation across intestinal epithelial cells is facilitated by ganglioside-like lipooligosaccharide structures. Infect Immun. 2012;80:3307-18.

15. Habib I, Louwen R, Uyttendaele M, Houf K, Vandenberg O, Nieuwenhuis EE, et al. Correlation between genotypic diversity, lipooligosaccharide gene locus class variation, and caco-2 cell invasion potential of Campylobacter jejuni isolates from chicken meat and humans: contribution to virulotyping Appl Environ Microbiol. 2009;75:4277-88.

16. Hardy CG, Lackey LG, Cannon J, Price LB, Silbergeld EK. Prevalence of potentially neuropathic Campylobacter jejuni strains on commercial broiler chicken products. Int J Food Microbiol. 2011;145:395-9.

17. Muraoka WT, Zhang Q. Phenotypic and genotypic evidence for L-fucose utilization by Campylobacter jejuni. J Bacteriol. 2011;193:1065-75.

18. Stahl M, Friis LM, Nothaft H, Liu X, Li J, Szymanski CM, et al. L-fucose utilization provides Campylobacter jejuni with a competitive advantage. Proc Natl Acad Sci U S A. 2011;108:7194-9.

19. Hansson I, Forshell LP, Gustafsson P, Boqvist S, Lindblad J, Engvall EO, et al. Summary of the Swedish Campylobacter program in broilers, 2001 through 2005. J Food Prot. 2007:70:2008-14

20. On SL, Holmes B. Assessment of enzyme detection tests useful in identification of campylobacteria. J Clin Microbiol. 1992;30:746-9.

21. Skirrow MB, Benjamin J. Differentiation of enteropathogenic Campylobacter. J Clin Pathol. 1980;33:1122.

22. Denis M, Soumet C, Rivoal K, Ermel G, Blivet D, Salvat G, et al. Development of a m-PCR assay for simultaneous identification of Campylobacter jejuni and C. coli. Lett Appl Microbiol. 1999;29:406-10.

23. Gonzalez I, Grant KA, Richardson PT, Park SF, Collins MD. Specific identification of the enteropathogens Campylobacter jejuni and Campylobacter coli by using a PCR test based on the ceuE gene encoding a putative virulence determinant. J Clin Microbiol. 1997;35:759-63.

24. Linton D, Owen RJ, Stanley J. Rapid identification by PCR of the genus Campylobacter and of five Campylobacter species enteropathogenic for man and animals. Res Microbiol. 1996;147:707-18.

25. Datta S, Niwa H, Itoh K. Prevalence of 11 pathogenic genes of Campylobacter jejuni by PCR in strains isolated from humans, poultry meat and broiler and bovine faeces. J Med Microbiol. 2003:52:345-8.

\section{Submit your next manuscript to BioMed Central and we will help you at every step:}

- We accept pre-submission inquiries

- Our selector tool helps you to find the most relevant journal

- We provide round the clock customer support

- Convenient online submission

- Thorough peer review

- Inclusion in PubMed and all major indexing services

- Maximum visibility for your research

Submit your manuscript at www.biomedcentral.com/submit 